\title{
Iatrogenic Hyponatremic Seizures After Routine Pelvic Ultrasonic Imaging
}

\author{
Sribari Gopal, MD, and Alan Blum, MD
}

We report the unusual case of a woman who suffered grand mal seizures in the emergency department after ingesting approximately $6 \mathrm{~L}$ of water in the course of preparing for a pelvic ultrasound examination. The cause appears to have been due to dilutional hyponatremia, despite normal renal function, caused by the rapid ingestion of a large volume of water. We believe the practice of encouraging patients to drink a large quantity of water to distend the bladder ought to be more precisely communicated.

\section{Case Report}

A 58-year-old postmenopausal schoolteacher came to her primary care physician's office after a weeklong history of vaginal spotting. After physical examination, Papanicolaou smear, and endometrial biopsy, her family physician referred her for a pelvic ultrasound examination.

Her medical history included hypertension and chronic constipation. As a college student, the patient had self-induced vomiting to lose weight, but she denied any episodes of bingeing and purging for the last 30 years. The patient was not preoccupied with her weight and did not have an altered body image. The patient admitted to checking the locks on her doors numerous times a day, but she had never sought medical care for obsessive and compulsive tendencies.

Her surgical history included two dilatation and curettage procedures and a hemorrhoidectomy. The patient had smoked one pack of cigarettes a day for 30 years but stopped smoking 5 years before. She denied the use of alcoholic beverages or illicit drugs, and she did not exercise regularly. Her

Submitted, revised, 20 October 1999.

From the Department of Family and Community Medicine (SG), Baylor College of Medicine, Houston, and the Department of Family Medicine (AB), College of Community Health Sciences, University of Alabama School of Medicine, Tuscaloosa. Address reprint requests to Alan Blum, MD, Department of Family Medicine, University of Alabama School of Medicine, PO Box 870374, Tuscaloosa, AL 35401 . family history included a myocardial infarction in her father and arthritis, colon cancer, diabetes, and hypertension in various distant relatives. Her medications included $30 \mathrm{mg}$ of nisoldipine once daily, $400 \mathrm{IU}$ of vitamin $\mathrm{E}$ daily, a multivitamin tablet once daily, and 1 square of phenolphthalein every 2 to 3 days as needed for constipation. The patient stated that she was allergic to codeine.

Before arriving at the radiology suite, the patient had received telephone instructions from the radiology technician to drink several liters of water. After an initial scan of her pelvis, the radiology technician noticed that the bladder was not distended fully, and the patient was encouraged to drink several more liters of water. After drinking approximately 3 more liters of water within 1 hour, the patient became drowsy, disoriented, and nauseated, and she vomited. The patient was escorted to the emergency department for further evaluation.

On initial examination, the patient was a thin, anxious-appearing woman in mild distress. She was alert to person and place but not to time. The patient's blood pressure was $159 / 97 \mathrm{~mm} \mathrm{Hg}$, her pulse was 89 beats per minute and regular, her weight was $42 \mathrm{~kg}$, her body mass index was 16.5 , and her respiratory rate was $22 / \mathrm{min}$ with an oxygen saturation of $100 \%$ on room air. Findings during an examination of her head, eyes, ears, nose, and throat were normal. Her pupils were $3 \mathrm{~mm}$ bilaterally and reactive to light and accommodation. There was no papilledema or other funduscopic abnormality. There were no thyroid nodules or thyromegaly. Her chest was clear to auscultation bilaterally with a prolonged expiratory phase. She had normal $S_{1}$ and $S_{2}$ with no audible murmurs, rubs, or gallops. No masses or axillary adenopathy were discovered on a breast examination. Her abdomen was soft, nontender, and not distended, with normoactive bowel sounds. A rectal examination was negative for blood in the stool. There was no hepatosplenomegaly. Her extremities showed no clubbing, cyanosis, or edema. Her skin had no 
Table 1. Laboratory Values.

\begin{tabular}{|c|c|c|c|c|c|c|c|}
\hline Laboratory Test & $2-9-98$ & $6-3-98$ & $6-4-98$ & $6-5-98$ & $6-6-98$ & $6-7-98$ & $6-8-98$ \\
\hline Calcium (mg/dL) & 10.4 & 8.1 & 7.5 & 7.7 & 7.8 & & 8.9 \\
\hline Chloride $(\mathrm{mEq} / \mathrm{L})$ & 86 & 98 & 86 & 96 & 106 & 108 & \\
\hline Creatine kinase $M B$ fraction (\%) & & $445 / 130.3$ & $2159 / 260.9$ & $16329 / 0.1$ & $29020 / 0.0$ & $32599 / 0.0$ & \\
\hline Creatinine $(\mathrm{mg} / \mathrm{dL})$ & 0.7 & 0.7 & 0.5 & 0.7 & 0.6 & 0.5 & \\
\hline Hematocrit (\%) & & 39.7 & 34.7 & 33.9 & & & \\
\hline Hemoglobin $(\mathrm{g} / \mathrm{dL})$ & & 13.7 & 12.6 & 12.1 & & & \\
\hline Magnesium (mg/dL) & & 1.3 & 2.3 & 2.0 & & 1.5 & \\
\hline Phosphate (mg/dL) & & 2.4 & 2.5 & 1.8 & & 2.5 & 3.6 \\
\hline Platelets $\left(\times 10^{3} / \mu \mathrm{L}\right)$ & & 245 & 214 & 231 & & & \\
\hline Potassium $(\mathrm{mEq} / \mathrm{L})$ & 4.8 & 2.7 & 3.7 & 4.4 & 3.7 & 3.8 & \\
\hline Serum osmolality $(\mathrm{mOsm} / \mathrm{kg})$ & & 242 & & & & & 284 \\
\hline Sodium $(\mathrm{mEq} / \mathrm{L})$ & 142 & 118 & & & & & \\
\hline Troponin I (mg/mL) & & 0.6 & & & & & \\
\hline Urine chloride (mEq/24 h) & & & & & & 135 & \\
\hline Urine potassium $(\mathrm{mEq} / 24 \mathrm{~h})$ & & & & & & 18 & \\
\hline Urine sodium $(\mathrm{mEq} / 24 \mathrm{~h})$ & & 119 & & & & 134 & \\
\hline Urine osmolality $(\mathrm{mOsm} / \mathrm{kg}$ ) & & 382 & & & & 338 & \\
\hline White blood cell count $\left(\times 10^{3} / \mu \mathrm{L}\right)$ & & 13.6 & 9.6 & 8.8 & & & \\
\hline
\end{tabular}

petechiae, purpura, or other rash. Cranial nerves II through XII were intact, as was upper and lower extremity strength. Deep tendon reflexes were $1+$ bilaterally in the patella, biceps tendon, and Achilles tendon. Her gait was unsteady and slightly wide based.

The patient was given $25 \mathrm{mg}$ of promethazine intramuscularly for her nausea and vomiting. While being reevaluated approximately 1 hour after administration of promethazine, she was observed to have two consecutive grand mal tonicclonic seizures. She was resuscitated with $0.9 \%$ saline, intravenous diazepam, and oxygen. An electrocardiogram showed pronounced $U$ waves, flattened $T$ waves, and 1-mm ST segment depression in leads $V_{4}$ and $V_{5}$.

Laboratory values showed profound electrolyte derangements (Table 1), notably serum sodium $(118 \mathrm{mEq} / \mathrm{L})$, serum potassium $(2.7 \mathrm{mEq} / \mathrm{L})$, and serum calcium $(8.1 \mathrm{mg} / \mathrm{dL})$. A computed tomogram (CT) of the head showed an old lacunar infarct of the right basal ganglia and age-appropriate degenerative changes. The patient was transferred to the intensive care unit for further treatment.

While in the intensive care unit, the patient had two more grand mal seizures for which she received intravenous phenytoin. The following laboratory tests were found to be within normal limits: thyroid-stimulating hormone, thyroxine, free thyrox- ine index, ferritin, transferrin, troponin I, creatine kinase-MB fraction, urine toxicology screening results, urine protoporphyrins, urine quantitative porphobilinogen deaminase, and urine myoglobin. Acute intermittent porphyria, diabetes insipidus, lung neoplasm, bulimia nervosa, chronic laxative abuse, subarachnoid hemorrhage, and inappropriate secretion of antidiuretic hormone (SIADH) were among the initial diagnoses under consideration.

A serum chemistry panel on a blood sample drawn in her primary physician's office (Table 1) 3 months earlier showed her serum potassium to be $4.8 \mathrm{mEq} / \mathrm{L}$ and serum sodium to be $142 \mathrm{mEq} / \mathrm{L}$.

A chest radiograph performed in the hospital showed a solitary $1-\mathrm{cm}$ pulmonary nodule in the right middle lobe, which was compared with radiographs taken 3 years earlier and found to be unchanged. Findings from magnetic resonance imaging of the brain and an electroencephalogram were normal. She was later found to be suffering from the syndrome of inappropriate antidiuretic hormone secretion (SIADH) because of an elevated urine osmolality (382 mOsm/L) compared with plasma osmolality $(242 \mathrm{mOsm} / \mathrm{L})$ in the face of normal levels of circulating blood plasma. The SIADH resolved after appropriate water restriction, which was discontinued after the patient was discharged from the hospital on the fifth hospital day. 
She was later found to have an obstructive pattern on spirometry consistent with chronic obstructive pulmonary disease. Subsequent CT scans of her abdomen and chest were performed. The solitary nodule in the middle lobe of the right lung was consistent with a hemangioma. A CT of the abdomen was normal. The patient has received care for 1 year as an outpatient and has been free of symptoms. The cause of her acute SIADH has not been established but is presumed to be due to her water intoxication during her pelvic ultrasound examination.

\section{Discussion}

There is a complex series of events involved in a normal renal response to a water load. The main determinant of how easily a person can tolerate a water load is the glomerular filtration rate (GFR). Water is first absorbed by the gastrointestinal tract, which increases circulating intravascular volume. The excess volume is detected by baroreceptors in the right atrium, and the decreased plasma osmolality is detected by osmoreceptors. Ultimately a cascade of events occurs (alterations of the feedback loops in production of atrial natriuretic factor, aldosterone, and renin) that ultimately decrease the production of antidiuretic hormone. This decrease of antidiuretic hormone increases the permeability of the distal nephron collecting tubules to water. This decrease in permeability to water promotes a diuresis. $^{1}$

SIADH is caused by an inappropriate concentration of urine secondary to a myriad of reported causes, including cancerous metastases to the brain, primary brain neoplasm, lung neoplasm or other mass, skull fracture, subarachnoid hemorrhage, porphyria, hypothyroidism, and tuberculosis. There are many different medications that can cause SIADH, most notably amitriptyline, chlorpropamide, carbamazepine, haloperidol, thiazide diuretics, thioridazine, vincristine, vinblastine, cyclophosphamide and even nicotine. $^{2}$

SIADH is diagnosed by hyponatremia and elevated urine osmolality when compared with serum osmolality in a patient with normal levels of circulating blood plasma. The standard treatment of SIADH is water restriction and attention to underlying cause. For patients who cannot tolerate water restriction, demeclocycline has been used. Its "mechanism of action is inhibition of the renal action of antidiuretic hormone.
We doubt that our patient had chronic underlying SIADH because her serum electrolytes were normal when measured in the office 4 months earlier. Review of the literature found no cases of hyponatremic seizures induced by drinking large quantities of water during an ultrasound examination. A case of induced hyponatremia was recently described, however, as a result of a combination of water ingestion and irrigation into the bladder during uroflowmetry. ${ }^{3}$ (Dilutional hyponatremia after cystoscopy or hysteroscopy has been well described in the urologic and gynecologic literature. ${ }^{4,5}$ )

There has been a similarly reported case of profound hyponatremia in a scientologist undergoing "purification" by ingesting large amounts of water and intense exercise in a sauna. ${ }^{6}$ A death was reported in a person who had undergone gastric lavage with large amounts of free water for suspected phosphorus poisoning. ${ }^{7}$ Hyponatremia has been documented in psychotic patients with polydipsia. ${ }^{8}$ There are also several reported cases of fatality secondary to psychogenic water intoxication. ${ }^{9,10}$

The routine recommendation to ingest a large quantity of water before a pelvic ultrasound examination seems unlikely to cause harm. Nonetheless, our experience with this patient suggests that to diminish the chance of an adverse event, physicians, radiologists, and other health care personnel should entertain a measure of caution. Through either a miscommunication on the part of health care personnel or a misunderstanding on the part of the patient, the patient in this report consumed several times the amount of water necessary to assure sufficient distention of the bladder for pelvic ultrasound examination. Based on our discussions with radiologists at several different medical centers, we would advise that patients be instructed in writing by the referring physician to drink $32 \mathrm{oz}$ of water (1 quart or $0.946 \mathrm{~L}) 1$ hour before the procedure.

\section{References}

1. Guyton AC. Textbook of medical physiology, ed 8. Philadelphia: Saunders, 1991:308-9.

2. AMA drug evaluations. Littleton, Mass: American Medical Association, 1991:710.

3. Issa MM, Pruthi RS, Vial C, McNamara DE, Terris MK. An unusual complication following uroflowmetry: water intoxication resulting in hyponatremia and seizure. Urol Int 1997;59:129-30.

4. Hahn RG. Dilutional hyponatraemia following transurethral operation for clot retention. $\mathrm{Br} J$ Anaesth 1991;67:339-40. 
5. Shirk GJ, Kaigh J. The use of low-viscosity fluids for hysteroscopy. J Am Assoc Gynecol Laparosc 1994;2: 11-21.

6. al-Zaki T, Jolly BT. Severe hyponatremia after "purification." Ann Emerg Med 1997;29:194-5.

7. Chen X, Huang G. Autopsy case report of a rare acute iatrogenic water intoxication with a review of the literature. Forensic Sci Int 1995;76:27-34.
8. Hariprasad MK, Eisinger RP, Nadler IM, Padmanabhan CS, Nidus BD. Hyponatremia in psychogenic polydipsia. Arch Intern Med 1980;140:1639-42.

9. Blotcky MJ, Grossman I, Looney JG. Psychogenic water intoxication: a fatality. Tex Med 1980;76:58-9.

10. DiMaio VJ, DiMaio SJ. Fatal water intoxication in a case of psychogenic polydipsia. Forensic Sci 1980;25: $332-5$. 